\title{
INTELIGÊNCIA EMOCIONAL DO ENFERMEIRO NA ABORDAGEM AO DOENTE CRÍTICO: ESTUDO QUALITATIVO
}

Emotional intelligence of nurses in the approach to critical patients: qualitative study

Inteligencia emocional de los enfermeros en el abordaje del paciente crítico: estudio cualitativo

\author{
Liliana Sousa, ${ }^{*}$ Cláudia Pereira, ${ }^{* *}$ Ana Lopes, ${ }^{*}$ Mirandolina Faísca, ${ }^{* *}$ Tânia Fortuna, ${ }^{* * *}$ \\ Fernanda Príncipe, ${ }^{* * *}$ Liliana Mota, ${ }^{* * * * *}$
}

\section{RESUMO}

Enquadramento: a inteligência emocional (IE) é o conjunto de capacidades do indivíduo para identificar, gerir e entender as suas próprias emoções e de se auto motivar perante situações constrangedoras e geradoras de conflitos. Reflete as atitudes e comportamentos do profissional perante diferentes contextos tendo impacte na qualidade da sua atuação. Objetivos: descrever a IE dos enfermeiros na área do cuidado ao doente crítico e identificar as estratégias comportamentais utilizadas pelos mesmos. Metodologia: estudo qualitativo, descritivo e exploratório. A recolha de dados foi realizada através de um focus group com 8 enfermeiros peritos no cuidado ao doente crítico. Resultados: emergiram 6 categorias relativas à IE dos enfermeiros na área do cuidado ao doente crítico: profissionalismo, relações interpessoais, organização do serviço, partilha de emoções, gestão do trabalho e autocontrolo emocional. Conclusão: como estratégias utilizadas identificou-se a partilha de emoções entre pares, a gestão do tempo para debater as situações existentes durante o horário laboral, a capacidade de autocontrolo emocional, a adaptabilidade ao meio e a forma como se estabelece a relação com o cliente.

Palavras-chave: inteligência emocional; enfermeiras e enfermeiros; cuidados críticos

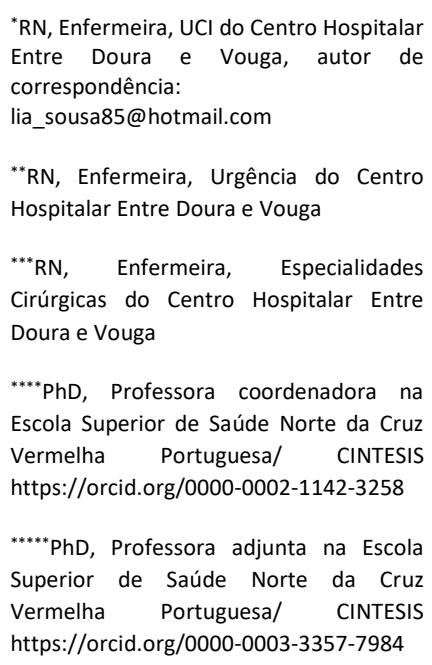

${ }^{* * * *} \mathrm{PhD}$, Professora coordenadora na Escola Superior de Saúde Norte da Cruz Vermelha Portuguesa/ CINTESIS https://orcid.org/0000-0002-1142-3258

${ }^{* * * * *}$ PhD, Professora adjunta na Escola Superior de Saúde Norte da Cruz Vermelha Portuguesa/ CINTESIS https://orcid.org/0000-0003-3357-7984

Como referenciar:

Sousa, L., Pereira, C., Lopes, A., Faísca, M., Fortuna, T., Príncipe, F., \& Mota, L. (2020). Inteligência emocional do enfermeiro na abordagem ao doente crítico: estudo qualitativo. Revista de Investigação \& Inovação em Saúde, vol.3(2). 39-48. doi.org/10.37914/riis.v3i2.97
Recebido para publicação em: 29/09/2020 Aceite para publicação em: 14/12/2020

\section{ABSTRACT}

Background: emotional intelligence (EI) is the individual's ability to identify emotions, manage and understand is own emotions and self-motivate in awkward and conflict situations. It reflects the professional's attitudes and behaviors in different contexts, impacting the quality of their performance. Objectives: to describe the IE of expert nurses in the critical care area and to identify the behavioral strategies used by nurses to approach the critically ill patient. Methodology: qualitative, descriptive and exploratory study. Data collection was performed using the focus group with 8 expert nurses in the critical care area. Results: there are 6 categories related to nurses IE in the area of critical patient care: professionalism, interpersonal relations, service organization, emotions sharing, work management and emotional self-control. Conclusion: the strategies identified were sharing emotions among peers, managing time to discuss existing situations during working hours, capacity of emotional selfcontrol, adaptability to the environment and the way to establish relationships with the client.

Keywords: emotional intelligence; nurses; critical care

\section{RESUMEN}

Encuadramiento: la inteligencia emocional (IE) es la capacidad del individuo para identificar emociones, manejar, comprender fenómenos emocionales y automotivarse en situaciones incómodas y problemáticas que generan conflictos intrínsecos y extrínsecos. Por lo tanto, refleja las actitudes y comportamientos de los profesionales en diferentes contextos, impactando la calidad de su desempeño. Objetivos: describir el IE de enfermeras expertas en el área de cuidados críticos e identificar las estrategias de comportamiento utilizadas por las enfermeras para abordar al paciente crítico. Metodología: este es un estudio cualitativo, descriptivo y exploratorio. La recolección de datos se realizó utilizando el grupo focal con 8 enfermeras expertas en el área de cuidados críticos. Resultados: hay 6 categorías relacionadas con el IE de las enfermeras en el área de atención crítica al paciente: profesionalismo, relaciones interpersonales, organización de servicios, intercambio de emociones, gestión del trabajo y autocontrol emocional. Conclusión: las estrategias identificadas fueron compartir emociones entre pares, administrar el tiempo para discutir situaciones durante las horas de trabajo, la capacidad individual para el autocontrol emocional, la adaptabilidad al medio ambiente, cómo establecer relaciones con el cliente y su autorregulación emocional.

Palabras clave: inteligencia emocional; enfermeras y enfermeros; cuidados críticos 
Inteligência emocional do enfermeiro na abordagem ao doente crítico: estudo qualitativo

\section{INTRODUÇÃO}

Sendo a enfermagem uma profissão caraterizada por elevada relação humana "é de extrema importância que os profissionais possuam capacidades de inteligência emocional (IE) para que possam orientar os seus pensamentos e ações. O enfermeiro deve ser capaz de controlar as suas emoções e compreender as emoções dos outros, pois só assim conseguirá atender às necessidades individuais do cliente" (Oliveira, 2019, p. 16). A IE dos enfermeiros tem impacte positivo nas atitudes, na adaptabilidade, melhoria nas relações interpessoais e maior orientação para valores positivos, retenção e prevenção do burnout (Nagel, Towell, Nel, \& Foxall, 2016).

Ao longo do tempo deu-se uma mudança de paradigma no setor do trabalho no que respeita às competências, pelo que hoje em dia, um dos grandes focos são as competências a nível emocional (Goleman, 2018).

IE é definida por Mayer, Salovey, \& Caruso (2004, p. 15) como "a capacidade de perceber acuradamente, de avaliar e de expressar emoções; a capacidade de perceber e/ou gerar sentimentos quando eles facilitam o pensamento; a capacidade de compreender a emoção e o conhecimento emocional e a capacidade de controlar emoções para promover o crescimento emocional e intelectual". A IE é a capacidade de compreender e gerir os pensamentos e sentimentos pessoais, bem como influenciar positivamente a comunicação interpessoal e bem-estar social (Benzo, Kirsch, Dulohery, \& Abascal-Bolado, 2016).

A IE dos enfermeiros tem potencial para a resolução de problemas, com impacte significativo nos resultados das intervenções implementadas nos doentes alvo dos seus cuidados (Lewis, 2019). É uma habilidade treinável devendo ser infundida nos curricula de enfermagem de forma a que os futuros profissionais de saúde melhorem as suas competências de IE (Lewis, 2019). A IE pode ser desenvolvida e aprendida em qualquer momento ou idade, e os programas de treinamento devem estar associados a maior bemestar e melhor regulação emocional (Benzo, Kirsch, Dulohery, \& Abascal-Bolado, 2016).

$\mathrm{Na}$ prática profissional cresce a necessidade de implementar programas de promoção da IE mais explícitos de forma a que os enfermeiros desenvolvam competências que permitam lidar de forma adequada na abordagem ao doente crítico a fim de desenvolverem estratégias de gestão emocional. Denota-se a falta de estudos a nível nacional nesta área envolvendo os profissionais de enfermagem, sendo por isso pertinente o desenvolvimento da investigação nesta área a fim de conhecer e produzir conhecimento que possa fundamentar a prática diária.

Com este estudo pretende-se descrever a IE dos enfermeiros na área do cuidado ao doente crítico e identificar as estratégias comportamentais utilizadas pelos mesmos.

\section{ENQUADRAMENTO/FUNDAMENTAÇÃO TEÓRICA}

Oliveira (2019) afirma que as emoções estão presentes na rotina de todos os seres humanos, sendo fundamental o seu controlo para o desenvolvimento pessoal. No cenário mundial é exigido que o indivíduo possua capacidades de IE que vão muito além de capacidades de inteligência cognitiva. É preciso saber controlar emoções para viver num contexto onde há constantes mudanças e desafios.

O cuidado ao doente crítico é descrito como cuidados de saúde prestados em ambiente tecnologicamente 


\section{Inteligência emocional do enfermeiro na abordagem ao doente crítico: estudo qualitativo}

avançado, que requer dos enfermeiros uma prática de enfermagem avançada com competências clínicas e tecnológicas que respondam aos processos complexos de saúde/doença da pessoa em situação crítica (Nagel, Towell, Nel, \& Foxall, 2016).

No estudo exploratório de Costa \& Faria (2009) acerca da IE no contexto de Enfermagem é salientada a necessidade dos profissionais de enfermagem terem mais oportunidades para refletir sobre as suas práticas através de formações nas áreas da IE, que forneçam possibilidades de treino em situações complexas e mais exigentes do ponto de vista da competência emocional.

Encarnação, Soares, \& Carvalho $(2018$, p. 8) concluíram que "IE influencia a forma como os enfermeiros desenvolvem a sua atividade. Esta é afetada pelas próprias caraterísticas individuais (...) e influencia o enfermeiro a nível pessoal, profissional e no cuidar." Reforçam ainda que a IE deve ser estimulada em contextos formativos.

A enfermagem como profissão deverá ter como alicerces fundamentais o conhecimento, capacidade, habilidade e atitudes necessárias para compreender, expressar e regular de forma apropriada os eventos emocionais. Contudo, no decorrer do exercício profissional os enfermeiros deparam-se com situações problemáticas, constrangedoras e geradoras de conflitos intrínsecos e extrínsecos. Exemplo representativo desta problemática é a complexidade inerente do cuidado ao doente crítico e toda a envolvência tecnológica do meio (Nagel, Towell, Nell, \& Foxall, 2016).

Os conceitos que compõe a IE enquadram-se nos padrões de conhecimento de enfermagem (Chinn, \& Kramer, 2018). Associado ao padrão de enfermagem conhecimento pessoal emerge a autoconsciência, como uma habilidade essencial em IE. Para a autorregulação das emoções é fundamental identificar e reconhecer as emoções em si mesmas enquanto contributo para uma relação terapêutica eficaz com o doente crítico (Lewis, 2019). O reconhecimento da forma como a pessoa expressa as suas emoções sobre o que considera certo, mesmo que os outros discordem, é componente fundamental da autoconfiança (Lewis, 2019). Estes factos alinham com os padrões éticos e emancipatórios dos padrões de conhecimento de enfermagem (Chinn, \& Kramer, 2018), enquanto processo de desenvolvimento do enfermeiro para a identificação da injustiça e desigualdade humanas na escolha do caminho correto.

\section{METODOLOGIA}

Trata-se de um estudo de caráter qualitativo, de tipo descritivo e exploratório, com recurso à realização de um focus group como meio de recolha de dados. A partir de uma amostra não probabilística e por conveniência, participaram no estudo 8 enfermeiros peritos na área do cuidado ao doente crítico, de diferentes hospitais da região norte de centro de Portugal.

Na seleção dos participantes foram considerados como critérios de inclusão ser enfermeiro a executar funções no Serviço de Urgência (4) ou no Serviço de Medicina Intensiva Polivalente (4) há pelo menos 10 anos.

A recolha de dados decorreu em julho de 2019. O guião do focus group foi constituído por questões abertas de forma a facilitar a expressão livre dos participantes.

Foi realizada gravação áudio de 50 minutos do focus group, sendo este posteriormente transcrito num documento de texto com recurso ao software 


\section{Inteligência emocional do enfermeiro na abordagem ao doente crítico: estudo qualitativo}

Microsoft Word e realizada uma codificação dos participantes (sujeito E1, sujeito E2...) de forma a garantir o anonimato. Foi realizada categorização à posteriori a partir do verbatim dos participantes de acordo com Bardin (2015).

Os procedimentos éticos foram garantidos, sendo previamente fornecida toda a informação acerca do estudo. Foi também garantida a confidencialidade da informação e o anonimato dos participantes sendo fornecido um termo de consentimento informado, assegurando se a participação livre e esclarecida a cada participante sem prejuízo para o mesmo caso pretenda a qualquer momento abandonar o estudo. $\mathrm{O}$ estudo teve parecer favorável da Comissão de ética (Parecer 010/2019)

\section{RESULTADOS/DISCUSSÃO}

A amostra é constituída por 8 enfermeiros, sendo que $6(80 \%)$ são do género feminino. A idade dos participantes varia entre 33 e 46 anos de idade, com uma média de idades de $39,75( \pm 4,60)$ anos. 0 tempo de exercício profissional varia entre os 10 a 25 anos com uma média de 15,37( $\pm 5,10)$ anos.

Aquando da análise dos dados resultantes do focus group com os participantes foram identificadas seis categorias: Profissionalismo, Relações Interpessoais, Organização do Serviço, Partilha de Emoções, Gestão do Trabalho e Autocontrolo Emocional.

\section{Profissionalismo}

O profissionalismo integra um momento de promoção da competência emocional, pois aqueles que apresentam a capacidade de identificar emoções, de as utilizar no processo de pensamento e de as compreender e analisar, sabendo lidar com elas relativamente a si próprio e aos outros, são indivíduos emocionalmente inteligentes. A competência emocional resulta da auto perceção da eficácia no controlo das situações.

"Tentar organizar, tentei ser eficiente, tentei ser eficaz, tentei não dar más respostas..." (E7, julho, 2019)

“A parte mais complicada foi separar a parte profissional do pessoal". (E6, julho, 2019)

“Por muito que tentemos ser profissionais há uma parte de nós que leva sempre um doente ou outro para casa". (E3, julho, 2019) Os participantes evidenciam que o reconhecimento das emoções e a autoconsciência das capacidades emocionais se refletem no desempenho profissional. Goleman (2019) advoga que é impossível separar a racionalidade das emoções, sendo estas que fundamentam o sentido da eficácia das decisões a partir do seu controlo.

\section{Relações Interpessoais}

As relações interpessoais inserem um pensamento focado na determinação do resultado pela qualidade nos relacionamentos desenvolvidos no âmbito do espaço de trabalho. Pode se dizer que nas relações estabelecidas entre os indivíduos está, em grande parte, a satisfação no trabalho realizado.

"Se a equipa não está a funcionar vai potencializar ainda mais o mau funcionamento em termos de gestão emocional e da equipa, nota se o stress." (E4, julho, 2019)

"Sei que todos os dias vai haver um conflito, más respostas entre os diferentes profissionais." (E4, julho, 2019)

"Conseguir discutir as coisas no momento ou logo a seguir ao momento de uma forma eficaz". (E8, julho, 2019) No discurso dos participantes é demonstrado que as relações interpessoais são um fator preponderante na prática de enfermagem, emergindo as seguintes subcategorias: gestão de conflitos, trabalho em equipa e a experiência profissional.

Os conflitos estão presentes em toda a parte da nossa vida, quer seja pessoal como profissional. Deste facto, 


\section{Inteligência emocional do enfermeiro na abordagem ao doente crítico: estudo qualitativo}

ressalta a importância da gestão de conflitos, a qual deve ser efetuada de forma correta, de modo a proporcionar resultados construtivos nas organizações. Conforme Beck (2009, p. 21), “além do diagnóstico bem feito do conflito é necessário que, ao lidar com conflitos, a pessoa tenha habilidades de atuação, as quais nem sempre são desenvolvidas na educação sistemática, em ambiente escolar ou em programas de desenvolvimento profissional".

"Se formos para o trabalho com a nossa vida organizada, com a nossa mente organizada é muito mais fácil gerir todos os conflitos." (E2, julho, 2019)

"Conseguir gerir os conflitos nos serviços, saber gerir a situação de forma que o teu trabalho fique harmonioso, saber gerir as nossas emoções..." (E2, julho, 2019)

"Consigam trabalhar todos e consigam comunicar sem haver conflitos". (E5, julho, 2019)

"Isto é um fator gerador de stress e conflitos, são capazes de estar lá uma hora à espera." (E6, julho, 2019)

Para Beck (2009, p. 14) "é importante lembrar que, embora o conflito seja determinado como uma situação negativa dentro das organizações, ocasionado principalmente por falha de comunicação e competições de interesses pessoais, os conceitos modernos mostram que este fenómeno também pode ser explorado para encorajar a autocrítica, a criatividade e a propensão para a aceitação de mudança".

Os participantes mostraram ser prática diária o trabalho em equipa, o qual se assume como imperativo uma vez que permite responder às exigências atuais em termos de cuidados de saúde e permite uma prática com padrões de qualidade mais elevados.

"Trabalhar para um fim conjunto." (E5, julho, 2019)

"Forma como a equipa se dá, a confiança que há nuns elementos e nos outros, cooperação." (E6, julho, 2019)
"Ter confiança com as pessoas com quem trabalhamos." (E3, julho, 2019)

Um fator referenciado pelos participantes e de relevo é a experiência profissional.

"a competência e experiência profissional também acho que ajuda muito a lidar com as emoções." (E8, julho, 2019)

"Um chefe de equipa com competências que é capaz de gerir mil e uma situações o serviço consegue funcionar muitas vezes com muitos conflitos." (E6, julho, 2019)

No contexto da prática de enfermagem hospitalar, o principal ator das equipas é o enfermeiro-chefe.

\section{Organização do Serviço}

A forma como as instituições estão organizadas quer do ponto de vista físico bem como na articulação entre os diferentes serviços e grupos de trabalho é influenciador no desempenho diário e satisfação dos profissionais, com impacte na IE. Os participantes relatam que o trabalho bem organizado e disciplinado é fundamental bem como a organização do espaço físico do serviço.

"da estrutura, dos recursos humanos, e assim toda a gente saiba que estamos neste modelo." (E4, julho, 2019) Para Barros (2009), a adoção do conceito e da prática do profissional/equipa de "referência", implica, além de mudanças na organização da equipa, o desenvolvimento de um novo olhar sobre o processo de cuidado, que deve envolver todos os recursos disponíveis e ampliar a disponibilidade para estar e fazer junto. Sugere que reuniões em equipa sejam a base da organização dos serviços e que devem contemplar espaços para discussão de casos e decisões conjuntas. Para os participantes a organização do serviço deve ter em linha de conta a cultura organizacional da instituição, as dotações e considerar os riscos de saúde profissionais.

Schein (2009, p. 16), define cultura organizacional como "um conjunto de representações e ideias 


\section{Inteligência emocional do enfermeiro na abordagem ao doente crítico: estudo qualitativo}

propostas por um líder e, quando validada sua aceitação, é ensinada aos novos membros do grupo a maneira correta de pensar e agir".

A equipa tem de estar preparada para a tomada de decisões independentes e interdependentes, sob o comando único de um líder, que transmita segurança e confiança. Os participantes manifestam-se sobre esse assunto:

"Vocês têm falta de enfermeiros, estão dispersos e as pessoas estão a acabar de chegar da rua." (E8, julho, 2019)

"Tivemos uma formação sobre gestão de stress, tivemos uma formação com a psicóloga do hospital e técnicas de relaxamento."

(E8, julho, 2019)

A necessidade de adequar os recursos de enfermagem tendo em conta a segurança, o nível de necessidade de cuidados de enfermagem dos clientes, a qualidade dos cuidados de enfermagem, a carga de trabalho, o ambiente de trabalho e o nível de qualificação/experiência dos enfermeiros, fez emergir a subcategoria dotação segura em enfermagem. Entende-se dotação, como sendo a quantidade e diferenciação necessária dos profissionais para a prestação de cuidados.

Cordeiro (2009) acerca da responsabilidade profissional, recursos humanos e qualidade dos cuidados em enfermagem reforça que os enfermeiros, mesmo quando existem em dotações insuficientes, não sentem que a sua responsabilidade diminua nos cuidados que prestam e optam por realizar os cuidados mais urgentes, priorizando as intervenções de acordo com esse critério e com as rotinas instituídas. Os participantes manifestam diferentes opiniões no que toca às dotações, o trabalho/serviço é o core para esta diferença.
“Nós temos dotações seguras na carga de horário, o trabalho também não é uma coisa exagerada, estamos num ambiente mais resguardado." (E3, julho, 2019)

"Falta de resposta das instituições nas dotações seguras, como no horário com o tempo descanso adequado, estratégias de relaxamento ou de formação em termos de stress reflete-se muito num dado que ninguém valoriza." (E3, julho, 2019) A enfermagem é uma profissão que inevitavelmente acarreta riscos saúde profissionais, segundo Santos \& Almeida (2016, p. 1) "os profissionais de saúde executam inúmeras tarefas sujeitas a fatores de risco/ riscos muito variados; contudo, se alguns são facilmente percetíveis para a maioria, outros são ignorados."

Os participantes defendem que as condições de trabalho devem garantir dotações seguras, horários adequados com tempo de descanso, desta forma diminuindo riscos profissionais.

"O absentismo implica que alguém trabalhe mais um bocado que já não está em condições, essa pessoa anda em linhas de burnout, isto aqui acaba por ser descontrolo total da gestão emocional não só pessoal." (E4, julho, 2019)

"Estão focadas para a produtividade da empresa e não para evitar taxas de suicídio profissionais." (E5, julho, 2019)

\section{Partilha de emoções}

Na partilha social das emoções verifica-se a partilha de experiências emocionais positivas e de experiências emocionais negativas. Relativamente ao impacte das experiências emocionais negativas, observa-se que estas experiências mantêm o impacte negativo, devido às cognições que envolvem a memória que origina a emoção. As cognições despoletam a ruminação mental, os pensamentos intrusivos e as imagens, aumentando a necessidade de falar e a partilha das emoções (Rimé, 2009). O grupo de participantes considera relevante a partilha de emoções no meio profissional entre pares, destacando-a como uma 


\section{Inteligência emocional do enfermeiro na abordagem ao doente crítico: estudo qualitativo}

estratégia que visa melhorar a IE na área do cuidado ao doente crítico.

"Momentos de partilha, na maior parte não temos tempo para

isso e as vezes nas passagens de turno, nos momentos mais calmos que as equipas partilham. Porque esta necessidade não é só da enfermagem é de toda a equipa multidisciplinar." (E6, julho,

"Não se tira tempo para se falar daquilo que nós vivemos a trabalhar e daquilo que levamos para casa todos os dias inevitavelmente." (E2, julho, 2019)

Crispim (2015, p. 42) defende que "a partilha social das emoções é uma forma mais especifica de regulação emocional que depende exclusivamente das relações sociais. Deste modo, as pessoas utilizam estas estratégias de forma mais ou menos intensa e mais ou menos eficaz e estas diferenças individuais determinam o modo como as pessoas se relacionam, adotando uma postura de maior evitamento ou proximidade na relação com os outros".

\section{Gestão do trabalho}

Goleman (2018) refere que a capacidade individual de cada profissional em gerir o seu trabalho/função contribuiu para a satisfação do ponto de vista profissional que também se reflete a nível pessoal e na sua IE. Diogo (2017, p. 21) refere que “...os enfermeiros ficam mais expostos ao stress físico e emocional e podem ter que se distanciar de tais emoções durante os cuidados".

"O stress que resulta de tu andares continuamente a alertar toda a gente para estas situações, andas a fazer o teu trabalho e o dos outros." (E6, julho, 2019)

"Muitas horas faz muita pressão, são muitas responsabilidades, muitas frustrações." (E7, julho, 2019)

"Não tenho tempo vamos despachar, e isto neste momento é umas das coisas que me está a trazer algum stress para casa." (E2,

julho, 2019)

Desta forma, identifica-se como estratégia a necessidade de uma melhor gestão do tempo, de forma a criar a possibilidade de debate das situações existentes durante o horário laboral.

\section{Autocontrolo Emocional}

Os enfermeiros enfrentam diariamente vários desafios na prática profissional, desafios que implicam esforço emocional e obrigam ao uso de habilidades interpessoais e emocionais. Deste modo, emerge a necessidade de saber lidar com os seus próprios sentimentos bem como as emoções que possam identificar no próximo.

International Council of Nurses (2016, p. 42) define autocontrolo como sendo "disposições tomadas para cuidar do necessário para a sua própria manutenção; para se conservar ativo; lidar com as suas necessidades individuais básicas e íntimas e as atividades da vida diária". Este define o conceito de emoção (201, p. 56) como "processo psicológico: sentimentos conscientes ou subconscientes, agradáveis ou dolorosos, expressos ou não expressados; podem aumentar com o stress ou com a doença".

Segundo Goleman (2019, p. 60) pessoas "autoconscientes do seu estado de espírito à medida que eles ocorrem, estas pessoas têm compreensivelmente alguma sofisticação nas suas vidas emocionais. A clareza com que analisam as suas próprias emoções pode sublinhar outros traços da personalidade: são autónomas e seguras dos seus próprios limites, têm uma boa saúde psicológica e tendem a encarar a vida de uma maneira mais positiva". Dada a complexidade do cuidado ao doente crítico, Goleman (2018, p. 31) preconiza que "quanto mais complexo for o trabalho, tanto mais a IE importa uma vez que uma deficiência nessas capacidades pode prejudicar a utilização de quaisquer perícias técnicas ou intelectuais que uma pessoa possa possuir". 


\section{Inteligência emocional do enfermeiro na abordagem ao doente crítico: estudo qualitativo}

“... termos a capacidade para sabermos que não conseguimos mais!" (E1, julho, 2019)

“...cada um por si tem de fazer a sua própria gestão para o seu bem-estar." (E7, julho, 2019)

“Pessoas felizes são mais produtivas...” (E5, julho, 2019)

No processo de Autocontrolo Emocional em contexto de atuação ao doente crítico, são identificadas como caraterísticas necessárias a capacidade de adaptação, a relação com o cliente e a autorregulação emocional, sendo estas caraterísticas as estratégias comportamentais identificadas ao longo deste estudo. International Council of Nurses (2016, p. 38) define adaptação como sendo "coping: gerir novas emoções." Esta definição vai ao encontro da teoria de Goleman (2018, p. 82) “durante a emergência, o cérebro retoma rotinas e respostas simples altamente familiares e põe de lado o pensamento complexo, a profundidade criativa e a planificação a longo prazo. A prioridade vai para o presente urgente ou a crise do dia" (...). Quando a mente está calma, as funções da memória de trabalho estão no seu máximo. Mas quando há uma emergência, o cérebro passa por um modo auto protetor, retirando recursos à memória de trabalho e atribuindo-os a outros locais do cérebro para manter os sentidos em estado de alerta máximo - uma postura mental destinada à sobrevivência".

Os participantes referem a necessidade que sentem em centrar-se no presente, na execução de técnicas a fim de se adaptarem à situação em causa e para serem capazes de dar resposta às necessidades exigidas pela profissão.

“Na sala de emergência queremos eficácia, queremos naquele momento centrar-nos no momento presente". (E5, julho, 2019) “Descarregar à posteriori, no momento o que nos interessa é ter a situação controlada, ter tudo controlado!" (E6, julho, 2019) "Pensar que estás a executar uma técnica e abstrair-te do resto..." (E3, julho, 2019)
Para Goleman (2018, p. 37) "as competências de que uma pessoa precisa para o êxito pode mudar à medida que ascende na carreira (...) no caso das melhores enfermeiras é o sentido de humor..."

“...nem que seja pelo humor..." (E7, julho, 2019)

A relação com o cliente adquire suma importância no palco do autocontrolo emocional, "A empatia exige calma e recetividade suficientes para que os subtis sinais dos sentimentos de outra pessoa possam ser recebidos e imitados pelo nosso próprio cérebro emocional" (Goleman, 2019, p. 117).

A relação que os enfermeiros desenvolvem com 0 doente é preponderante para o seu desempenho profissional, os participantes verbalizaram que 0 contexto em que desempenham as suas funções é influenciador na forma como estabelecem essa relação.

"A nível empático, em contexto de urgência, é completamente diferente que nos cuidados intensivos..." (E5, julho, 2019) "Nos cuidados intensivos o doente vai acordar e torna-se próximo, começa a fazer parte da nossa vida e marca-nos..." (E5, julho, 2019)

"Na UCIP tens mais contacto com a família, mais proximidade com a situação" (E5, julho, 2019)

Os participantes referem que a capacidade de autorregulação emocional é crucial para a performance profissional e da equipa, este fato não implica a ligação direta com o desempenho intelectual de cada um, como corrobora Goleman (2019).

“Há pessoas cognitivamente extremamente inteligentes e a nível de IE deixa muito a desejar." (E5, julho, 2019)

“Perante uma emoção de contrariedade, de raiva, reagem de uma forma extremamente agressiva dentro da equipa. Temos pessoas que menos inteligentes e perante uma contrariedade, uma situação de crise, são pessoas que sabem geri-la." (E5, julho,

2019)

A "perceção da forma como as nossas emoções afetam aquilo que fazemos constitui a competência emocional 


\section{Inteligência emocional do enfermeiro na abordagem ao doente crítico: estudo qualitativo}

fundamental. Quem não possui essa capacidade tornase vulnerável..." (Goleman, 2018, p. 62).

"Uma emoção de uma pessoa mal gerida dentro da equipa destabiliza completamente!" (E5, julho, 2019)

"Se soubermos exatamente que fizermos um bom trabalho temos mais paz interior quando deixamos o trabalho" (E8, julho,

A IE deve ser uma constante em desenvolvimento na vida dos enfermeiros, mas sabemos que essa não é uma realidade. Atualmente a enfermagem está a passar por momentos de dificuldade no país, a sobrecarga de trabalho, o acumular de horas extra, os rácios elevados de clientes nas unidades de saúde e baixo número de profissionais para prestar os cuidados, são algumas das dificuldades. É primordial que se faça uma reflexão de modo a dar mais atenção e apoio aos enfermeiros e, por isso, considera se importante adotar estratégias que contribuam para o desenvolvimento das capacidades de IE.

\section{CONCLUSÃO}

Da análise dos resultados foram identificadas seis categorias que ilustram a visão dos participantes na temática da gestão emocional no cuidar do doente crítico. Os participantes referem que o profissionalismo (saber separar o que é pessoal do que é profissional), a forma como se estabelecem as relações interpessoais (gestão de conflitos, o trabalho em equipa e experiência profissional) são fundamentais para conseguirem equilíbrio na gestão emocional.

A capacidade que cada um possui para gerir o tempo e o seu trabalho é preponderante na gestão de stress no decorrer do horário de trabalho e consequentemente na gestão da IE. É referido ainda pelos participantes que a forma como o serviço se encontra organizado influencia o seu desempenho dos profissionais, a cultura organizacional da instituição, a capacidade desta em manter as dotações seguras dos profissionais é crucial evitando os riscos de saúde dos mesmos.

As estratégias comportamentais utilizadas pelos enfermeiros na abordagem ao doente crítico e identificadas neste estudo, baseiam se em ultrapassar as dificuldades diárias e inerentes à profissão, a partilha de emoções entre pares, a necessidade de terem tempo para debaterem situações existentes durante o horário laboral, bem como a capacidade que cada um possui no autocontrolo emocional passando este pela adaptabilidade de cada um ao meio, a forma como estabelecem relações com o próprio cliente e a sua autorregulação emocional.

Tal como em outros estudos, este também apresenta limitações, tal como o défice de evidência na área científica de enfermagem.

O presente estudo servirá em termos futuros de base para um estudo de investigação ação na área do burnout bem como para o desenvolvimento de diagnósticos, intervenções e estratégias com vista a melhorar a gestão emocional dos enfermeiros na abordagem ao doente crítico.

Tendo em linha de conta esta realidade, consideramse benéfica a realização do estudo com vista à produção de conhecimento científico na área de Enfermagem. A divulgação dos resultados deste tornase essencial e promotora de ganhos em saúde nos cuidados ao doente crítico. Acreditamos na promoção da investigação e na articulação com a prática de enfermagem, de forma a dar visibilidade aos resultados. 


\section{REFERÊNCIAS BIBLIOGRÁFICAS}

Bardin, L. (2015). Análise de Conteúdo. Lisboa: Edições 70.

Barros, J. O. (2009). A contrução de projetos terapêuticos no campo da saúde mental: apontamentos acerca das novas tecnologias de cuidado. São Paulo: Universidade de São Paulo.

Beck, G. (2009). Conflito nas organizações. São Paulo: Centro Universitário Feevale.

Benzo, R. P., Kirsch, J. L., Dulohery, M. M., \& AbascalBolado, B. (2016). Emotional Intelligence: A Novel Outcome Associated with Wellbeing and SelfManagement in Chronic Obstructive Pulmonary Disease. Annals of the American Thoracic Society, 13(1), 10-16. https://doi.org/10.1513/AnnalsATS.201508-4900C

Cordeiro, A. (2009). Responsabilidade profissional: recursos humanos e qualidade dos cuidados de enfermagem. Dissertação de Mestrado: Faculdade de Medicina da Universidade de Lisboa.

Costa, A. C., \& Faria, L. (2009). A inteligência emocional no contexto de enfermagem: estudo exploratório com o questionário de competência emocional (QCE). Braga: Universidade do Minho.

Chinn, P. \& Kramer, M. (2018). Integrated Theory and Knowledge Development in Nursing. 8th ed. St Louis, MO: Elsevier Mosby.

Crispim, I. (2015). Estilos vinculação, regulação emocional e partilha social das emoções em estudantes universitários. Universidade Lusófona de Humanidades e Tecnologias.

Diogo, P. (2017). Relação Terapêutica e Emoções: Envolvimento versus Distanciamento Emocional dos Enfermeiros. Pensar Enfermagem.

Encarnaçao, R., Soares, E., \& Carvalho, A. (2018). Inteligência Emocional: Fatores Influenciadores e impacto nos enfermeiros em cuidados intensivos. Revista Rene, Vol. 19,33-58
Goleman, D. (2018). Trabalhar com Inteligência Emocional. Círculo de Leitores.

Goleman, D. (2019). Inteligência Emocional. Lisboa: Temas e debates.

International Council of Nurses. (2016). Classificação internacional para a prática de enfermagem: CIPE versão 2015. Lisboa, Portugal: Lusodidacta.

Lewis S. L. (2019). Emotional Intelligence in Neonatal Intensive Care Unit Nurses: Decreasing Moral Distress in End-of-Life Care and Laying a Foundation for Improved Outcomes: An Integrative Review. Journal of hospice and palliative nursing: JHPN: the official journal of the Hospice and Palliative Nurses Association, 21(4), 250-256. https://doi.org/10.1097/NJH.0000000000000561

Mayer, J., Salovey, P., \& Caruso, D. (2004). Emotional Inteligence: Theroy, findings and implications. Psychological Inquiry, 15(3), 197-215

Nagel, Y., Towell, A., Nel, E., \& Foxall, F. (2016). The emotional intelligence of registered nurses commencing critical care nursing. Curationis, 39(1), e1-e7. https://doi.org/10.4102/curationis.v39i1.1606

International Council of Nurses. (2015). CIPE - Versão 2015: classificação internacional para a prática da enfermagem. Lisboa: Ordem dos Enfermeiros.

Oliveira, K. S. (2019). Inteligência Emocional dos Enfermeiros: contributos da supervisão clínica. Tese de Mestrado: Escola Superior de Saúde do Porto.

Rimé, B. (2009). Emotion elicits the social sharing of emotion: Theory and empirical review. Emotion Review, 1(1), 60-85

Santos, M., \& Almeida, A. (2016). Profissionais de Saúde: principais riscos e fatores de risco, eventuais doenças profissionais e medidas de proteção recomendadas. Revista Portuguesa de Saúde Ocupacional, 28-52. DOI:10.31252/RPSO.26.10.2016

Schein, E. (2009). Cultura organizacional e Liderança. São Paulo: Atlas. 